\title{
Self-doping effects on the morphology, electrochemical and conductivity properties of self-assembled polyanilines
}

\author{
Rong-Ho Lee ${ }^{\mathrm{a}, *}$, Hung-Hsiang Lai ${ }^{\mathrm{a}}$, Jiunn-Jye Wang ${ }^{\mathrm{a}}$, Ru-Jong Jeng ${ }^{\mathrm{b}}$, Jiang-Jen Lin ${ }^{\mathrm{c}}$ \\ a Department of Chemical and Material Engineering, National Yunlin University of Science E Technology, Yunlin, Taiwan, ROC \\ b Department of Chemical Engineering, National Chung Hsing University, Taichung, Taiwan, ROC \\ ${ }^{c}$ Institute of Polymer Science and Engineering, National Taiwan University, Taipei, Taiwan, ROC
}

\section{A R T I C L E I N F O}

\section{Article history:}

Received 26 November 2007

Received in revised form 4 June 2008

Accepted 19 June 2008

Available online 28 June 2008

\section{Keywords:}

Conducting polymer

Polyaniline

Self-assembly

Nanostructure

\begin{abstract}
A B S T R A C T
Copolymerizations of a self-doping monomer (o-aminobenzenesulfonic acid, SAN) and aniline (AN) in different molar ratios via the self-assembly process were conducted to prepare self-doping polyanilines (SPANIs). The morphology of SPANIs can be changed from microspheres and nanotube to coral reef like structures by simply increasing the molar ratio of the monomer (AN) to the dopant (SAN). The relationship among the AN to SAN molar ratio, the morphology, UV-Vis absorption behaviors, thermal stability, electrochemical behavior, crystalline density, and conductivity of self-doped PANIs are investigated. The nanostructures were strongly dependent on the AN to SAN mole ratio. UV-Vis absorption behaviors changed with increasing degrees of self-doping, which correspond to the various SPANI redox states. Moreover, the decomposition temperature was also relative to the degree of self-doping. A higher degree of self-doping led to higher SPANI degradation temperatures. Oxidation and reduction current peaks occur at more positive potentials for SPANIs with a higher degree of self-doping. High crystalline density led to the high room temperature conductivity of the SPANIs, which was attributed to the interaction between the polymer chains.
\end{abstract}

(c) 2008 Elsevier B.V. All rights reserved.

\section{Introduction}

Conducting polymers have recently received much attention due to their long conjugation length, metallic conductivity, and their promise for applications in molecular wires, nano-electronics, optoelectronic devices, and biomedical devices [1-3]. Researchers have extensively studied nanostructure conducting polymers, including polypyrrole [4,5], polyalkyl-thiophene [6,7], and polyaniline (PANI) [8-21]. PANI has been studied more than the other two conducting polymers because it is relatively cheap, easy to prepare, exhibits unique chemical and physical properties, and has good thermal and environmental stabilities [14,15]. The conductivity and physical properties of polymers are affected by the PANI nanostructure. Several studies have synthesized PANIs with various nanostructures, such as microporous structures, hollow microspheres, and nanotubes [8-10,21]. Researchers have used different organic acids as dopants for preparing PANI with various nanostructures [8-10]. On the other hand, synthesizing PANI in the presence of protonic acid as a self-doping monomer, such as 2-aminonaphthalene-4,8-disulfonic acid, and 0 -aminobenzenesulfonic acid (SAN), has also attracted a great deal of interest in recent years [16-21]. In comparison with the external acid doped PANI method [8-10], a self-doping monomer does

\footnotetext{
* Corresponding author. Tel.: +886 5 5342601x4625; fax: +886 55312071. E-mail address: lerongho@yuntech.edu.tw (R.-H. Lee).
}

not need to be removed after PANI polymerization because the dopant also plays the roles of surfactant and comonomer of PANI [16-21].

The nanostructure and morphology of conducting polymers play important roles in determining material properties and their potential applications in technology. Conducting polymers with hollow spheres hold promise for application in the medicine and catalyst fields [8]. Nanotube and nanowire structures are favorable for application in optoelectronic nano-devices [8-10,21]. External acid doped PANIs with nanotube structure prepared by the self-assembly process have been widely studied by several research groups [8-10]. Zhang et al. reported that PANIs with nanostructures varying from hollow spheres to nanotubes could be produced by simply changing the dopant to monomer molar ratio [8]. Zhang et al. also investigated the hydrogen bonding effect on self-assembled PANI nanostructures [9]. A third study by Zhang et al. reported that highly crystalline PANI nanotubes could be produced by dicarboxylic acid doping [10]. More recently, sulfonated PANIs with different degrees of sulfonation (or doping) were produced by the self-assembly process in the presence of SAN as a self-doping monomer [21]. Yang et al. discussed the nanofiber structure formation mechanism of self-doped PANI (SPANI) [21].

According to previous studies, most self-doping sulfonated PANIs were produced by the electrochemical synthesis method $[17,19,20]$. These studies reported on the electrochemical properties and conductivity of sulfonated PANIs. However, sulfonated PANIs morphologies have not been taken before $[17,19,20]$. Researchers have also investigated the copolymerizations of a self-doping 
monomer SAN and aniline (AN) in different mole ratios via selfassembly process as a method for preparing nanotube structure SPANIs [21]. This paper studies in detail the synthetic condition effects on nanotube diameter, such as the AN/SAN molar ratio, monomer concentration, oxidant concentration, reaction temperature and time. Nevertheless, the discussion of AN to SAN mole ratio effects on nanostructures only ranged from 0.5 to $4 \mathrm{M}$. This paper does not examine the influence of the degree of sulfonation or doping on thermal stability, electrochemical properties, and crystalline density. Therefore, the preparation of self-doping PANIs with different nanostructures was conducted by changing the AN/SAN molar ratio from 1 to 8 . The doping degrees of SPANIs were obtained using an elemental analyzer. The relationship between the molar ratio of AN to SAN, the morphology, UV-Vis absorption behaviors, thermal stability, electrochemical property, crystalline density, and conductivity of the self-doping PANIs are all discussed.

\section{Experimental details}

\subsection{Synthesis of self-doped polyaniline}

As Scheme 1 indicates, the SPANIs were synthesized by the selfassembly method [21]. The reaction conditions of various SPANIs are summarizes in Table 1. For example, AN $(0.083 \mathrm{M})$ and SAN $(0.083 \mathrm{M})$ in different molar ratios $(1: 1)$ were stirred and dissolved in distilled water under $4{ }^{\circ} \mathrm{C}$. An oxidant ammonium peroxydisulfate (APS; $0.4 \mathrm{M}$ ) aqueous solution was then added to the AN-SAN mixture solution and stirred for $3 \mathrm{~min}$. SPANI polymerization occurred under a stationary condition under $4{ }^{\circ} \mathrm{C}$ for two days. A dark green solid sheet of SPANI SP1 was removed from the reaction solution and thoroughly rinsed with $\mathrm{H}_{2} \mathrm{O}$ and $\mathrm{CH}_{3} \mathrm{OH}$, and then dried under vacuum for one day.

\subsection{Characterization of self-doped polyaniline}

Fourier-transform infrared spectra (FTIR) of SPANIs were recorded with a Perkin Elmer Spectrum One FTIR spectrometer. Elemental analysis was carried out with an elemental analyzer (Elementar Vario EL III). The UV-Vis spectra were measured using a Hewlett-Packard 8453 with a photodiode array detector. Thermogravimetric analysis (TGA) of the SPANIs was performed under a nitrogen atmosphere at a heating rate of $10{ }^{\circ} \mathrm{C} / \mathrm{min}$ with a Thermogravimetric Analyzer (TA Instruments, TGA-2050). The nanostructure of self-doping PANI was recorded by a cold field emission scanning electron microscope (FESEM; HITACHI S-4800; operating voltage: $1.5-3.0 \mathrm{kV}$ ). The morphology was measured by a high-resolution transmission electron microscope (HRTEM; JEOL JEM-2010) with an acceleration voltage of $45 \mathrm{kV}$. The redox potentials of the SPANIs were determined by a cyclic voltammetry using an AutoLav electrochemical analyzer with a scanning rate at $100 \mathrm{mV} / \mathrm{s}$. The redox potentials of the SPANI films coated on indium tin oxide glass were measured in $1 \mathrm{M}$ sulfonic acid electrolytic solution. It is important to note that the SPANI thin films were coated from the m-cresol solution of SPANI (1 wt.\%). The X-Ray diffraction patterns of SPANIs were taken by an X-Ray diffractometer

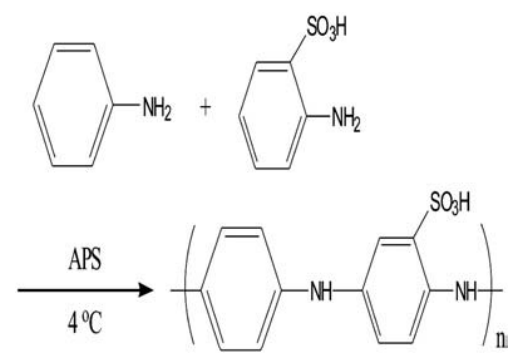

Scheme 1. Synthesis of self-doping polyaniline.
Table 1

Reaction conditions of the self-doped polyanilines

\begin{tabular}{llllll}
\hline SPANIs & $\begin{array}{l}\text { AN/SAN } \\
\text { molar ratio }\end{array}$ & $\begin{array}{l}\text { AN concentration } \\
(\mathrm{M})\end{array}$ & $\begin{array}{l}\text { APS concentration } \\
(\mathrm{M})\end{array}$ & $\begin{array}{l}\text { Reaction } \\
\text { Time }(\mathrm{h})\end{array}$ & $\begin{array}{l}\text { Sulfonation } \\
\text { degree }^{\mathrm{a}}(\%)\end{array}$ \\
\hline SP1 & 1 & 0.083 & 0.4 & 48 & 24.1 \\
SP2 & 4 & 0.092 & 0.4 & 24 & 11.8 \\
SP3 & 4 & 0.092 & 0.4 & 96 & 15.2 \\
SP4 & 8 & 0.184 & 0.4 & 96 & 9.1 \\
\hline
\end{tabular}

a Doping degree was obtained using the elemental analyzer.

(PANalytical X'Pert PRO MPO) with monochromatized CuKa (1.5406 ̊) radiation. The conductivity of SPANI pellets at room temperature was measured by a conductance meter (ELCHEMA Model CM-308) at a constant voltage of $500 \mathrm{mV}$.

\section{Results and discussion}

\subsection{Chemical structure characterization of SPANIs}

To prove whether or not the SPANIs were produced by copolymerization, FTIR spectroscopy was used to determine the presence of $\mathrm{SO}^{-}$groups attached to the aromatic rings. Fig. 1 shows the FT-IR spectra of SPANIs SP1-SP4. The characteristic peaks around 1580 and $1480 \mathrm{~cm}^{-1}$ correspond to the stretching of the benzenoid and quinoid units in SPANIs, respectively [22]. The absorption peaks at 1300 and $1246 \mathrm{~cm}^{-1}$ represent the $\mathrm{C}-\mathrm{H}$ stretching vibration with aromatic conjugation [23]. The peak at $1040 \mathrm{~cm}^{-1}$ is caused by $\mathrm{S}=0$ stretching, confirming the presence of sulfonate in PANIs [24]. The S-O stretching peak is at $700 \mathrm{~cm}^{-1}$, while the peak at $630 \mathrm{~cm}^{-1}$ represents the $\mathrm{C}-\mathrm{S}$ stretching vibration. The absorption peaks at 1040,700 , and $630 \mathrm{~cm}^{-1}$ in the FTIR spectra of the SPANIs are consistent with the presence of the $-\mathrm{SO}^{-}$group attached to the aromatic rings [25]. The FTIR-spectra evidenced that the SPANIs were obtained by the copolymerization of AN and SAN monomers. In addition, the elemental compositions of SPANIs were obtained using an elemental analyzer to determine the degree of sulfonation or doping. Table 1 summarizes the SPANI degrees of sulfonation. The sulfonation degree was $24.1 \%$ for SPANI SP1 and $11.8 \%$ for SPANI SP2. Moreover, the sulfonation degree of SPANI SP3 was slightly higher than SPANI SP2, which corresponds to the increased reaction time. Compared to the other SPANIs (SP1-SP3), SPANI SP4 showed the lowest degree of sulfonation because it had the highest molar ratio of AN to SAN. Based on the above, the sulfonation degree of SPANIs was related to the AN/SAN molar ratio and reaction time of polymerization. Higher AN/SAN molar ratio led to a lower sulfonation degree, whereas the extention of reaction time was favorable for the enhanced sulfonation degree of SPANIs.

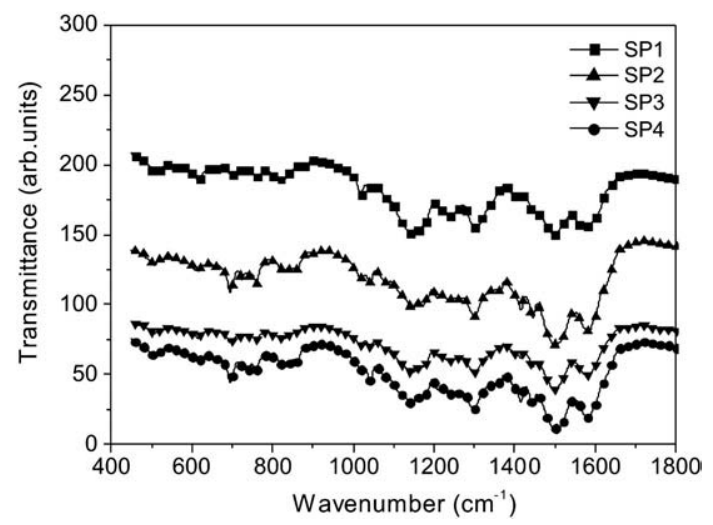

Fig. 1. FT-IR spectra of self-doping PANIs with different AN to SAN molar ratio. 


\subsection{Morphologies and formation mechanism of SPANIs}

SEM and TEM micrographs of SPANIs with different nanostructures are shown in Figs. 2 and 3. The SPANI SP1 material was prepared with an AN to SAN molar ratio of 1. Nanocluster structure of SPANI SP1 was evident in SEM images after polymerization occurred for $48 \mathrm{~h}$. TEM images show that the nanocluster structure was composed of hollow microspheres. The average outer and inner diameters of the hollow microspheres are about $420-600$ and $340-380 \mathrm{~nm}$, respectively. As the molar ratio of AN to SAN increased up to 4, the nanotube structure was observed for the assembled SPANIs (SP2-SP3). Moreover, the average outer diameter of nanotubes increased steadily with increasing reaction time. Figs. 2 and 3 illustrate that the average outer and inner diameters measured from the SEM and TEM images range from 83-185 and 13-36 nm, respectively. The length of the nanotubes is about $0.5-1.0 \mu \mathrm{m}$, independent of the reaction time. Furthermore, the SPANI nanotubes almost disappeared instead of forming a coral reef like SPANI for the SPANI SP4 with an AN to SAN molar ratio of 8. SPANI nanotube aggregation caused the coral reef like structure.

Based on the information above, Fig. 4 shows the proposed formation mechanisms of self-doping PANIs with different morphologies. In the polymerization process, SAN plays the roles of surfactant and monomer for the self-doping PANIs. First, SAN and aniline cations form micelles in aqueous solution. The aniline cations can appear in the interface of micelles and water. The polymerization initially occurs at the surface of the micelles as the oxidant APS is added [19,21,26,27]. In addition, the micelles tend to aggregate and react along the direction of the polymer chain in the polymerization process. The micelles act as the template in the self-assembly of SPANI to form microspheres or nanotube structures $[9,19,21,26,27]$. In a reaction solution with a low aniline content, the aniline cations can only react at the surface of the micelles to form hollow microspheres. As a result, the hollow microspheres could be clearly observed in the TEM images of SPANI SP1. Aggregation of the hollow microspheres led to the formation of the nano-cluster structure of SPANI. This is evidenced in the SEM image of SPANI SP1 (see Fig. 2(a)). Fig. 4 shows the proposed nanocluster structure. For the SPANIs SP2 and SP3, the materials were prepared with an AN to SAN molar ratio of 4. Low SAN content led to the formation of small diameter micelles in the aqueous solution. Large AN content caused micelles to react and join together along the direction of the polymer chain in the polymerization process. Therefore, SPANI nanotubes with a small inner diameter and long
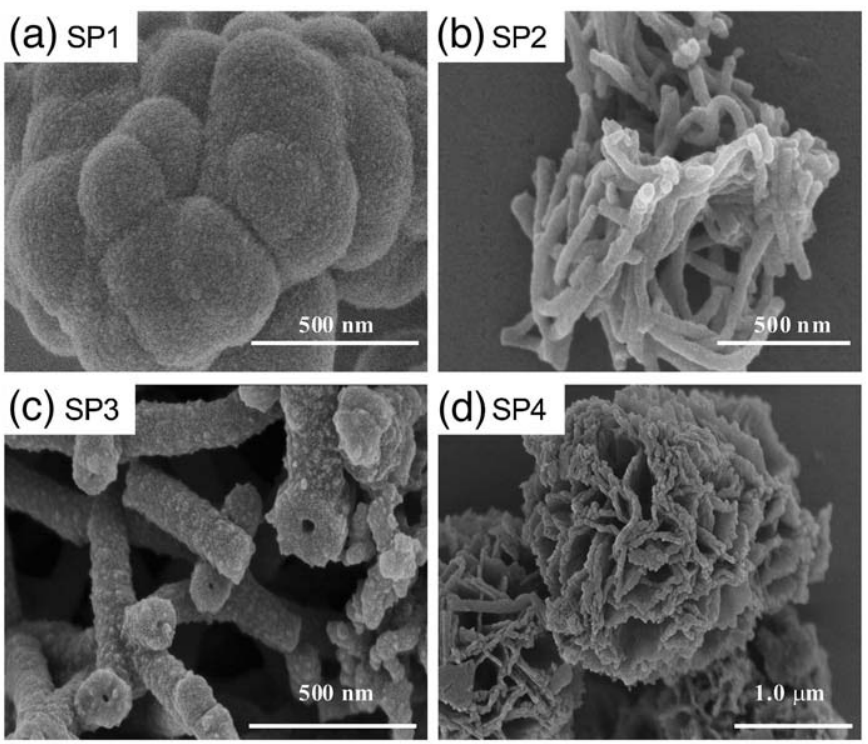

Fig. 2. SEM images of self-doping PANIs with different nanostructures.
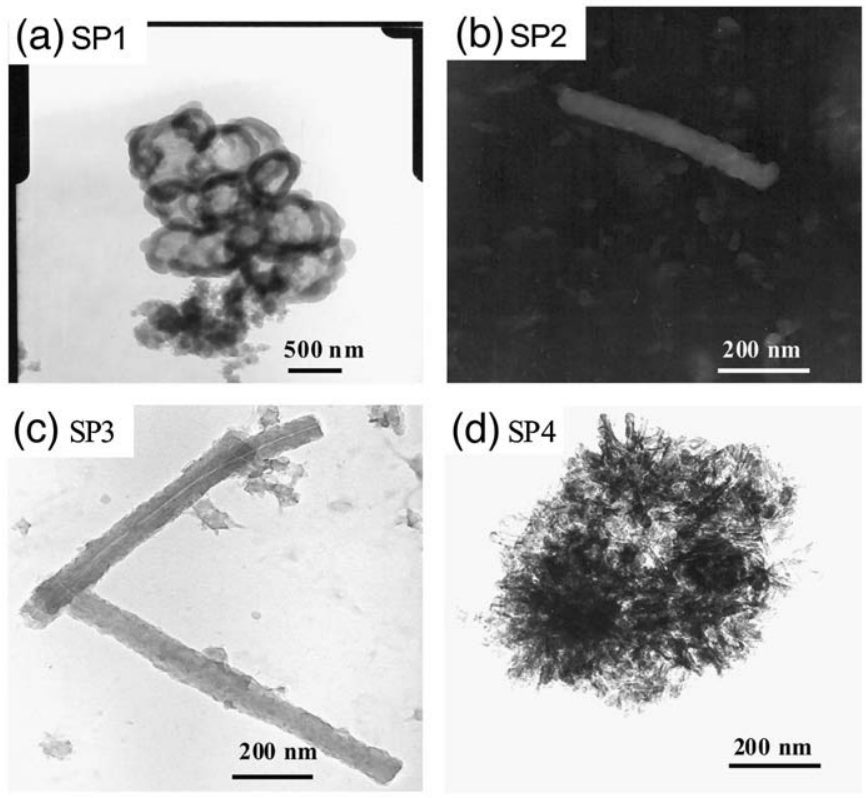

Fig. 3. TEM images of self-doping PANIs with different nanostructures.

length structure were observed for SPANIs SP2 and SP3. Fig. 4(b) shows the proposed nanotube structure. Nanotube length did not increase significantly as the reaction time increased from 24 to $96 \mathrm{~h}$, but increasing reaction time increased the average outer diameter of the nanotubes. The inner diameter was primarily determined by the
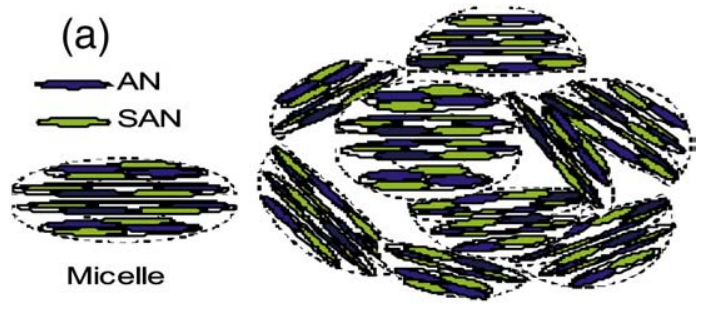

(b)
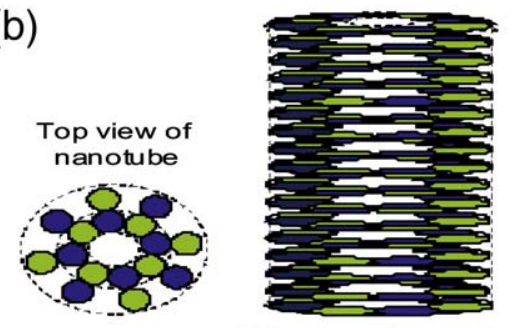

(c)

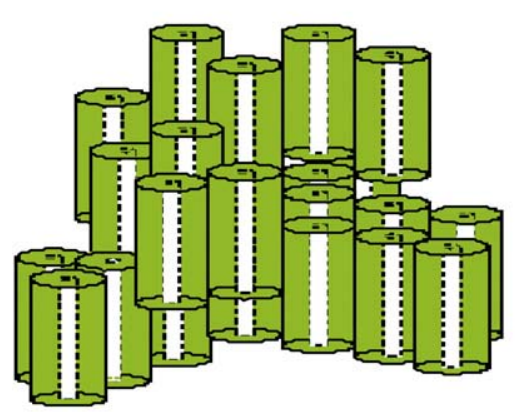

Aggregation of nanotube

Fig. 4. Proposed nanostructures of self-doping PANIs (a) nano-cluster model, (b) nanotube model, and (c) coral reef like model, respectively. 
size of the micelle in the initial reaction stage. Furthermore, strong interaction between the $-\mathrm{SO}^{-}$SAN group and the AN cationic radical nitrogen atoms in the polymer chains caused the SPANI nanotubes to aggregate together as the AN to SAN molar ratio increased up to 8. As a result, a coral reef like structure is apparent in SPANI SP4. This coral reef like structure is proposed as the aggregation of nanotubes, which is shown in Fig. 4(c). The SPANI morphology can be changed from microspheres to nanotubes, or even to coral reef like structures by simply changing the molar ratio of AN to SAN.

\subsection{UV-Vis absorption of SPANIS}

The UV-Vis absorption spectra of SPANIs are shown in Fig. 5. The first absorption band at around $300 \mathrm{~nm}$ was attributed to the reduced state (leucoemeraldine) of SPANIs. The results indicate that SPANI SP1 exhibits a higher absorption intensity than the other samples, which corresponds to the lowest aniline unit content in the polymer backbone. A low AN to SAN ratio and high degree of sulfonation was favorable to the reduced state formation in the backbone of self-doped PANI. For SPANIs SP2 and SP3, the intensity of the first absorption band increased as the reaction time increased, accomplished with increasing the reduced state content in the polymer chain. This might be due to the fact that the degree of sulfonation grew as the reaction time progressed. Strong interaction between SAN and AN was favorable for the reduced state formation in the SPANI backbone. Yang et al. reported the interaction between SPANI polymer chains [21]. In addition, the SPANI reduced state was also affected by the AN to SAN ratio. The reduced state content of SPANI SP4 was lower than for SPANI SP3, which corresponds to the enhanced AN to SAN ratio and reduced degree of sulfonation. The second absorption band, corresponding to the partial oxidation state of SPANIs, was observed at wavelength around $400 \mathrm{~nm}$. This partial oxidation state was assigned as the intermediate state between the leucoemeraldine form containing benzenoid rings and the emeraldine form containing conjugated quinoid rings in the main chain of the SPANI [21]. The second absorption band exhibited higher intensity for SPANI SP2-SP4, in comparison with SPANI SP1. This difference demonstrates that a decreasing degree of sulfonation is favorable to the formation of the intermediate state between leucoemeraldine and emeraldine forms. Moreover, the second absorption bands became broader as the reaction time increased for SPANIs SP2-SP4. This broadening implies that the SPANI redox state became more complex as the reaction progressed. In addition, the third absorption band corresponding to the fully oxidized pernigraniline form appeared at a wavelength near $800 \mathrm{~nm}$. SPANI SP1 exhibits the higher third band intensity than the other SPANIs. A low degree of sulfonation led to the decreased pernigraniline form $[21,28]$.

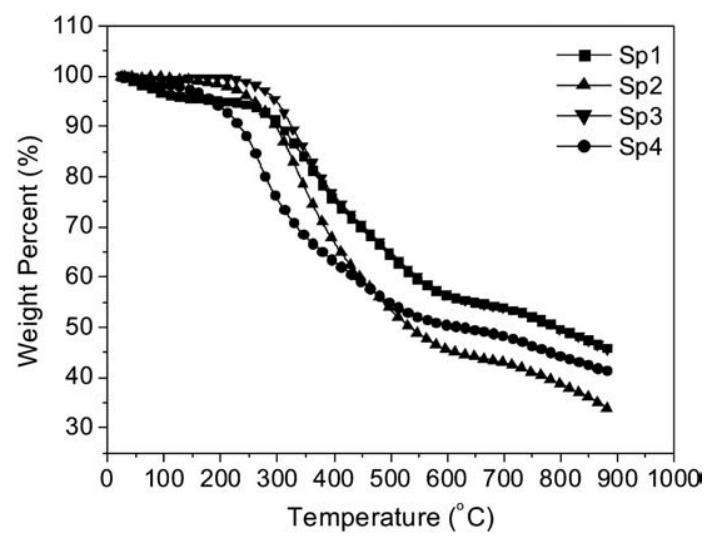

Fig. 6. TGA thermograms of SPANIs SP1-SP4.

\subsection{Thermal properties of SPANIS}

Fig. 6 shows the TGA thermograms of the SPANIs. A typical three step weight loss behavior was observed in the TGA curves of all SPANIs. Three weight loss steps included the loss of solvent or water, decomposition initiated by dopants, and decomposition of the SPANI chains $[29,30]$. For SPANI SP1, the weight loss at temperature above $350{ }^{\circ} \mathrm{C}$ was comparable to SPANI SP3 despite the fact that SPANI SP1 has a larger weight loss at temperature below $350{ }^{\circ} \mathrm{C}$ than SPANI SP3. Moreover, SP1 and SP3 exhibit better thermal stability than SP2 and SP4. The TGA curves demonstrate that the SPANI decomposition temperature was affected by the degree of doping or sulfonation. A higher degree of sulfonation led to a higher degradation temperature. This is attributed to the interaction between the SAN $-\mathrm{SO}^{-}$group and the AN cationic radical nitrogen atom.

\subsection{Electrochemical properties of SPANIs}

The electrochemical behaviors of the SPANI films obtained from $1 \mathrm{M} \mathrm{H}_{2} \mathrm{SO}_{4}$ electrolytic solution are shown in Fig. 7. Table 2 summarizes the potential of oxidation and reduction current peaks. The oxidation and reduction current peaks occur at more positive potentials for SPANIs SP1 and SP3 than for SPANIs SP2 and SP4. A higher degree of sulfonation led to a higher potential of oxidation and reduction current peaks for SPANIs SP1 and SP3. This is attributed to the fact that the decrease of the charge delocalization and difficulties in ion movement inside the polymer film, caused by the presence of the substituent groups. The electrochemical behaviors of the self-

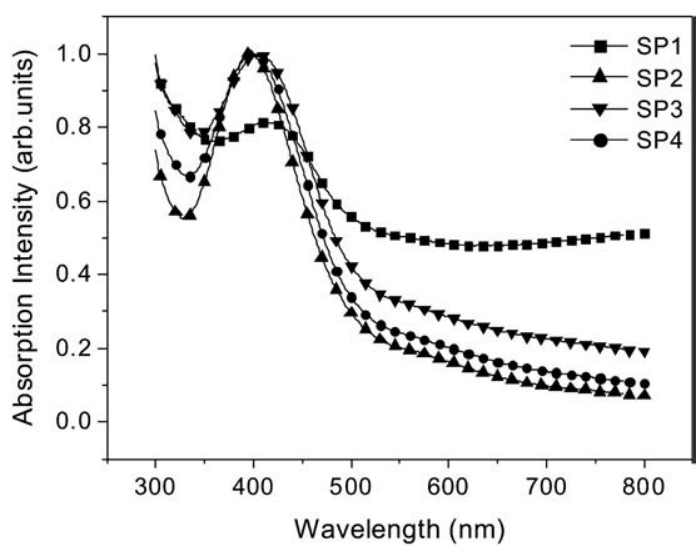

Fig. 5. UV-Vis absorption spectra of SPANIs SP1-SP4.

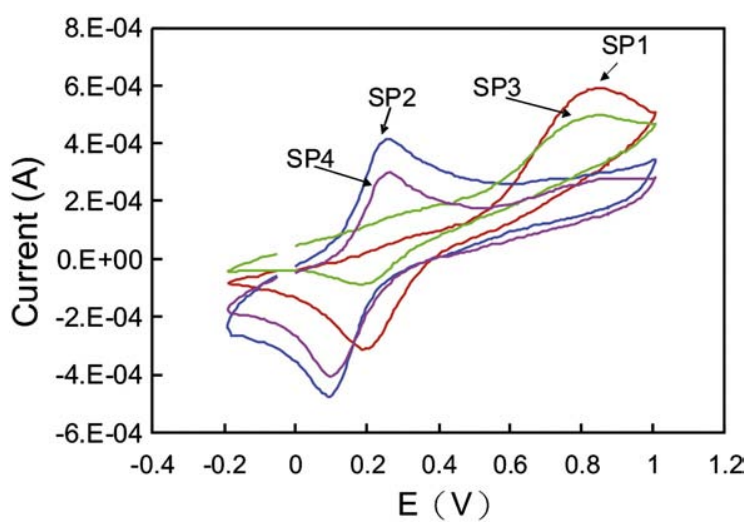

Fig. 7. Electrochemical behavior of the self-doping PANIs SP1-SP4. 
doping PANIs were strongly dependent on the degree of sulfonation of the polymer chains.

\subsection{X-Ray analysis of SPANIs}

Fig. 8 shows the X-Ray diffraction patterns of SPANIs SP1-SP4. For SPANI SP1, a broad peak centered at $2 \theta=22^{\circ}$ occurred for sample SP1 with an AN to SAN molar ratio of 1. For SPANIs SP2-SP4, several sharp peaks occurred at $2 \theta=6^{\circ}, 18^{\circ}, 19^{\circ}, 23^{\circ}, 26^{\circ}$, and $28^{\circ}$ corresponding to $d=14.7,4.9,4.4,3.86,3.42$, and $3.18 \AA$. The sharp peak at $2 \theta=6^{\circ}$ is attributed to the diffraction along periodicity parallel to the SPANI chain, while the others peaks may be caused by periodicity perpendicular to the SPANI chain [31,32]. The results indicate that the microsphere structure SPANI shows an amorphous morphology, while high crystalline morphology occurs in nanotube or coral reef like structure SPANIs.

\subsection{Conductivity of self-doped PANIs}

The AN to SAN mole ratio and reaction time effects on the room temperature conductivity of SPANIs are summarizes in Table 2 . Conductivity increased significantly from $8.5 \times 10^{-7}$ to $6.3 \times 10^{-4} \mathrm{~S} / \mathrm{cm}$ as the AN to SAN molar ratio increased from 1 to 8 . SPANI SP1 with the highest sulfonation degree exhibited a lowest conductivity than those of SPANI SP2-SP4. According to the literature, the conductivity values of the SPANIs are inversely related to the degree of sulfonation. The origin of the lower conductivity and greater electron-localization with a higher degree of sulfonation were attributed to several factors. One of the reasons is that the presence of the side substitute group led to the separation enhancement of polymer main-chains and reduced inter-chain diffusion of charge carriers, subsequently resulting in lower SPANI conductivity. Moreover, the interaction between the SAN ${ }_{-} \mathrm{SO}^{-}$group and the AN cationic radical nitrogen atoms is likely to force the polymer main chain out of planarity and lower the overlap of orbitals along the conjugated chains by twisting the aromatic rings relative to one another [33]. Consequently, the charge carrier's mobility both along the polymer main-chain and between polymer chains was reduced. Apart from that, the conductivity was also changed as the reaction time increased, corresponding to the change in SPANI morphology. The conductivity of SPANIs SP2 or SP3 with a nanotube structure is one to two orders of magnitude higher than the conductivity of SP1 with a microsphere structure. Moreover, SPANI SP3 shows a higher conductivity than SPANI SP2, which is presumably due to the enhancement of the polymer chain's packing density throughout the SP3 nanotube. In addition, the average conductivity of the SAN doped PANIs shows a higher value than that previously reported [21]. High crystalline density led to the high room temperature conductivity of the SPANIs. Furthermore, the conductivity of SPANI SP4 with a coral reef like structure was lower than the conductivity of SPANI SP3 with a nanotube structure. This difference may be due to the random aggregation of nanotubes in the coral reef like structure. This can be observed in the TEM image of SPANI SP4 (see Fig. 3(d)). Apart from that, the conductivity of the SAN doped PANIs with nanotube structure in this work was lower than the external organic acid doped PANIs [8-10]. Side substitute group results in the decrease of the charge carrier's mobility both along the

Table 2

Electro-chemical behaviors and room temperature conductivity of self-doped polyaniline films

\begin{tabular}{llll}
\hline SPANIs & Oxidation potential $(\mathrm{V})$ & Reduction potential $(\mathrm{V})$ & Film Conductivity $(\mathrm{S} / \mathrm{cm})$ \\
\hline SP1 & 0.86 & 0.19 & $8.5 \times 10^{-7}$ \\
SP2 & 0.26 & 0.10 & $1.03 \times 10^{-5}$ \\
SP3 & 0.86 & 0.18 & $6.30 \times 10^{-4}$ \\
SP4 & 0.26 & 0.10 & $2.44 \times 10^{-5}$ \\
\hline
\end{tabular}

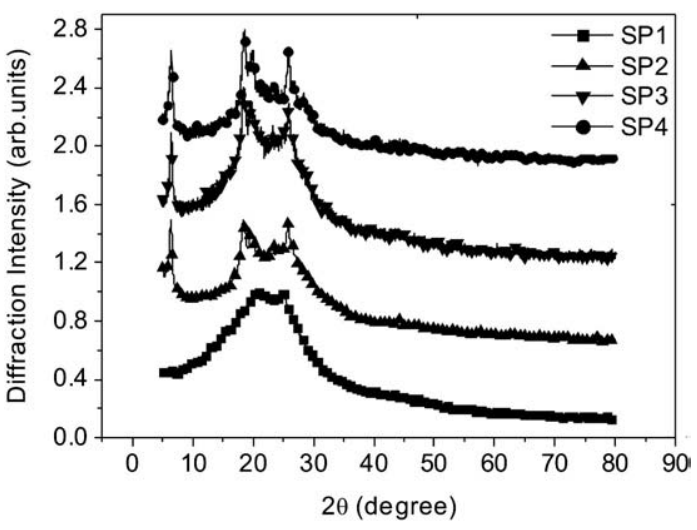

Fig. 8. X-Ray diffraction patterns of self-doping PANIs SP1-SP4.

polymer main-chain and between polymer chains, subsequently the lower conductivity of the self-doped PANIs was observed in comparison with external organic acid doped PANI.

\section{Conclusion}

The sulfonation degree effect on the morphology, UV-Vis absorption, thermal stability, electrochemical behavior, crystalline density, and conductivity property of self-assembled polyaniline was investigated. The morphology of self-doped PANI changed from nanoclusters and nanotubes to a coral reef like nanostructure by increasing the AN to SAN molar ratio in the copolymerization reaction. The coral reef like nanostructure has never before been observed in the SPANIs. The thermal stability, electrochemical behavior, crystalline density, and conductivity properties were strongly dependent on the degree of doping degree and the nanostructure of the SAN doped polyanilines. Different nanostructures, higher crystalline density, and larger conductivity have been achieved in this work.

\section{Acknowledgments}

The authors thank the National Science Council of Taiwan, ROC, for financial support (Grant NSC 96-2221-E-224-030). Helpful assistance in the measurement of electrochemical and conductivity properties from Prof. C. W. Lin is also acknowledged.

\section{References}

[1] J. Lei, V.P. Menon, C.R. Martin, Polym. Adv. Tech. 4 (1992) 124.

[2] M. Lemaire, D. Delabouglise, R. Garreau, A. Gay, J. Roncal, J. Chem. Soc., Chem. Commun. (1998) 695

[3] Z. Chen, Y. Takei, B.A. Deoye, T. Nagaoka, Analyst 125 (2000) 2249.

[4] F. Chen, P. Akhtar, A.P.K. Maguire, G.G. Wallace, Aust. J. Chem. 50 (1997) 939

[5] D. Delabouglise, F. Garnier, Synth. Met. 39 (1990) 117.

[6] D. Kotkar, V. Joshi, P.K. Ghosh, J. Chem. Soc., Chem. Commun. (1998) 917.

[7] M. Andersson, P.O. Ekeblad, T. Hjertberg, O. Wennerstrom, O. Inganas, Polym. Commun. 32 (1991) 546.

[8] L. Zhang, M. Wan, Adv. Funct. Mater. 13 (2003) 815

[9] L. Zhang, Y. Long, Z. Chen, M. Wan, Adv. Funct. Mater. 14 (2004) 693.

[10] Z. Zhang, M. Wan, Y. Wei, Adv. Funct. Mater. 16 (2006) 1100.

[11] M. Horie, I. Yamaguchi, T. Yamamoto, Macromolecules 39 (2006) 7493.

[12] N.A. Zaidi, J.P. Foreman, G. Tzamalis, S.C. Monkman, A.P. Monkman, Adv. Funct Mater. 14 (2004) 479.

[13] E.N. Konyushenko, J. Stejskal, I. Sedenkova, M. Trchova, I. Sapurina, M. Cieslar, J. Prokes, Polym. Int. 56 (2006) 31.

[14] Y. Chao, P. Smith, A.J. Hegger, Synth. Met. 48 (1992) 91.

[15] S.W. Ng, K.G. Neoh, J.T. Sampanthar, E.T. Kang, K.L. Tan, J. Phys. Chem., B 105 (2001) 5618.

[16] R. Mazeikiene, A. Malinauskas, Mater. Chem. Phys. 83 (2004) 184.

[17] X.H. Wang, J. Li, L.X. Wang, X.B. Jing, F.S. Wang, Synth. Met. 69 (1995) 147.

[18] H. Varela, S.L.A. Maranhao, R.M.Q. Mello, E.A. Ticianelli, R.M. Torresi, Synth. Met. 122 (2001) 321

[19] Y. Sahin, K. Pekmez, A. Yildiz, Synth. Met. 129 (2002) 107.

[20] A. Malinauskas, R. Holze, Electrochim. Acta 43 (1998) 521

[21] C.H. Yang, Y.K. Chih, H.E. Cheng, C.H. Chen, Polymer 46 (2005) 10688. 
[22] Y. Cao, S. Li, Z. Xue, D. Guo, Synth. Met. 16 (1986) 305.

[23] J. Tang, X. Jing, B. Wang, F. Wang, Synth. Met. 24 (1988) 231.

[24] J. Yue, A.J. Epstein, A.G. MacDiarmid, Mol. Cryst. Liq. Cryst. 189 (1990) 255

[25] J.H. Fuhrhop, W. Helfrich, Chem. Rev. 93 (1993) 1565

[26] M. Adachi, T. Harada, M. Harada, Langmuir 16 (2000) 2376

[27] B.J. Kim, S.G. Oh, M.G. Han, S.S. In, Langmuir 16 (2000) 5841.

[28] A.G. MacDiarmid, A.J. Epstein, Synth. Met. 65 (1994) 103.
[29] A. Boyle, J.F. Penneau, E. Genies, C. Riekel, J. Polym. Sci., Part B, Polym. Phys. 30 (1992) 265.

[30] S. Palaniappan, B.H. Narayana, J. Polym. Sci., Part A, Polym. Chem. 32 (1994) 2431.

[31] Y.B. Moon, Y. Cao, P. Smith, A.J. Heeger, Poym. Commun. 30 (1989) 196

[32] J. Huang, M.X. Wan, J. Polym. Sci., Part A, Polym. Chem. 37 (1999) 151.

[33] P.M. MacManus, R.J. Cushman, S.C. Yang, J. Phys. Chem. 91 (1987) 744. 